Jonathan O. Bauer*

\title{
The crystal structure of the triclinic polymorph of hexameric (trimethylsilyl)methyllithium, $\mathrm{C}_{24} \mathrm{H}_{66} \mathrm{Li}_{6} \mathrm{Si}_{6}$
}

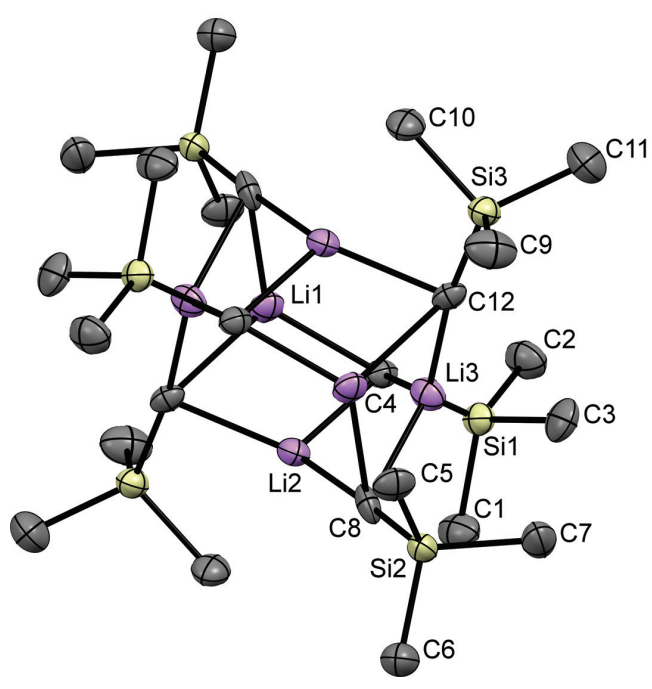

https://doi.org/10.1515/ncrs-2019-0662

Received September 7, 2019; accepted October 21, 2019; available online November 9, 2019

\section{Abstract}

$\mathrm{C}_{24} \mathrm{H}_{66} \mathrm{Li}_{6} \mathrm{Si}_{6}$, triclinic, $P \overline{1} \quad$ (no. 2), $a=12.2492(9) \AA$, $b=12.4617(9) \AA, \quad c=14.3841(11) \AA, \quad \alpha=74.189(6)$, $\beta=74.543(7), \quad \gamma=85.970(6), \quad V=2036.2(3) \AA^{3}, \quad Z=2$, $R_{\mathrm{gt}}(F)=0.0465, w R_{\text {ref }}\left(F^{2}\right)=0.0802, T=173(2) \mathrm{K}$.

\section{CCDC no.: 1960466}

The molecular structure of the title compound is shown in the figure, with the labeling scheme indicating the asymmetric unit. Tables 1 and 2 contain details of the measurement method and a list of the atoms including atomic coordinates and displacement parameters.

\footnotetext{
*Corresponding author: Jonathan 0. Bauer, Institut für Anorganische Chemie, Fakultät für Chemie und Pharmazie, Universität Regensburg, Universitätsstraße 31, D-93053 Regensburg, Germany, e-mail: jonathan.bauer@ur.de. https://orcid.org/0000-0002-95757430
}

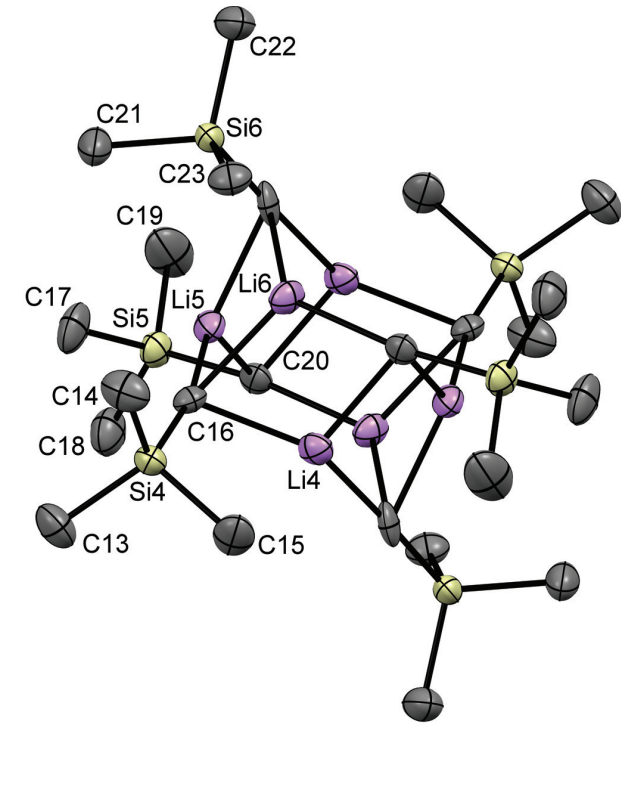

Table 1: Data collection and handling.
Crystal:

Size:

Wavelength:

$\mu$ :

Diffractometer, scan mode:

$\theta_{\max }$, completeness:

$N(h k l)_{\text {measured }}, N(h k l)_{\text {unique }}, R_{\text {int }}$ :

Criterion for $I_{\text {obs }}, N(h k l)_{\text {gt }}$ :

$N(\text { param })_{\text {refined: }}$

Programs:
Needle, colorless

$0.30 \times 0.20 \times 0.20 \mathrm{~mm}$

Mo $K \alpha$ radiation $(0.71073 \AA)$

$0.22 \mathrm{~mm}^{-1}$

Xcalibur S, $\omega$-scans

$26^{\circ},>99 \%$

13964, 7965, 0.049

$I_{\text {obs }}>2 \sigma\left(I_{\text {obs }}\right), 3837$

457

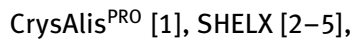

Mercury [6], publCIF [7], WinGX and ORTEP [8]

\section{Source of materials}

Single crystals of the title compound, suitable for X-ray structural analysis, were obtained from a concentrated solution of (trimethylsilyl)methyllithium in pentane at room temperature within one day.

\section{Experimental details}

Single-crystal X-ray diffraction analysis was performed on an Oxford Diffraction CCD Xcalibur S diffractometer [1] at 
Table 2: Fractional atomic coordinates and isotropic or equivalent isotropic displacement parameters $\left(\AA^{2}\right)$.

\begin{tabular}{|c|c|c|c|c|}
\hline Atom & $x$ & $y$ & $z$ & $U_{\text {iso }}{ }^{*} / U_{\text {eq }}$ \\
\hline Li1 & $0.3877(4)$ & $0.4387(4)$ & $0.4496(3)$ & $0.0293(11)$ \\
\hline Li2 & $0.5156(4)$ & $0.5994(4)$ & $0.3636(3)$ & $0.0291(12)$ \\
\hline Li3 & $0.4378(4)$ & $0.6542(4)$ & $0.5182(3)$ & $0.0306(12)$ \\
\hline Li4 & $0.1607(4)$ & $1.0592(4)$ & $0.9707(3)$ & $0.0303(12)$ \\
\hline Li5 & $0.0113(4)$ & $0.8632(4)$ & $0.9423(3)$ & $0.0303(12)$ \\
\hline Li6 & $0.0743(4)$ & $0.8843(4)$ & $1.0857(3)$ & $0.0310(12)$ \\
\hline Si1 & $0.25936(7)$ & $0.74690(6)$ & $0.43493(6)$ & $0.0278(2)$ \\
\hline $\mathrm{Si} 2$ & $0.70000(7)$ & $0.77965(6)$ & $0.46615(5)$ & $0.0264(2)$ \\
\hline $\mathrm{Si} 3$ & $0.44114(7)$ & $0.50122(6)$ & $0.80146(5)$ & $0.0279(2)$ \\
\hline $\mathrm{Si} 4$ & $0.34127(7)$ & $0.91510(6)$ & $0.90257(5)$ & $0.0276(2)$ \\
\hline Si5 & $-0.10647(7)$ & $0.86207(7)$ & $0.79800(6)$ & $0.0317(2)$ \\
\hline Si6 & $-0.00987(7)$ & $0.66224(6)$ & $1.16733(6)$ & $0.0282(2)$ \\
\hline C1 & $0.3178(2)$ & $0.8434(2)$ & $0.30893(19)$ & $0.0419(8)$ \\
\hline $\mathrm{H} 1 \mathrm{~A}$ & 0.399250 & 0.853446 & 0.298308 & $0.063^{\star}$ \\
\hline H1B & 0.279702 & 0.915676 & 0.304944 & $0.063^{\star}$ \\
\hline $\mathrm{H} 1 \mathrm{C}$ & 0.305140 & 0.811571 & 0.257394 & $0.063^{*}$ \\
\hline C2 & $0.1019(2)$ & $0.7422(2)$ & $0.4655(2)$ & $0.0436(8)$ \\
\hline $\mathrm{H} 2 \mathrm{~A}$ & 0.078588 & 0.706959 & 0.420674 & $0.065^{\star}$ \\
\hline $\mathrm{H} 2 \mathrm{~B}$ & 0.071861 & 0.818304 & 0.456997 & $0.065^{\star}$ \\
\hline $\mathrm{H} 2 \mathrm{C}$ & 0.072251 & 0.698988 & 0.534931 & $0.065^{\star}$ \\
\hline C3 & $0.2931(4)$ & $0.8091(3)$ & $0.5291(3)$ & $0.0439(10)$ \\
\hline $\mathrm{C} 4$ & $0.3289(3)$ & $0.6105(2)$ & $0.4400(2)$ & $0.0255(7)$ \\
\hline $\mathrm{C} 5$ & $0.7681(3)$ & $0.6788(3)$ & $0.5565(3)$ & $0.0376(9)$ \\
\hline C6 & $0.8178(2)$ & $0.8662(2)$ & $0.37040(19)$ & $0.0405(8)$ \\
\hline H6A & 0.872396 & 0.817726 & 0.337913 & $0.061^{\star}$ \\
\hline H6B & 0.855841 & 0.906206 & 0.402933 & $0.061^{*}$ \\
\hline $\mathrm{H} 6 \mathrm{C}$ & 0.787045 & 0.920024 & 0.320134 & $0.061^{\star}$ \\
\hline $\mathrm{C} 7$ & $0.6111(2)$ & $0.8756(2)$ & $0.5350(2)$ & $0.0434(9)$ \\
\hline H7A & 0.583389 & 0.936564 & 0.487731 & $0.065^{\star}$ \\
\hline H7B & 0.656651 & 0.906254 & 0.568349 & $0.065^{\star}$ \\
\hline $\mathrm{H} 7 \mathrm{C}$ & 0.546563 & 0.834245 & 0.585073 & $0.065^{\star}$ \\
\hline C8 & $0.6109(3)$ & $0.7014(3)$ & $0.4208(2)$ & $0.0294(8)$ \\
\hline C9 & $0.5722(2)$ & $0.5286(3)$ & $0.8330(2)$ & $0.0506(9)$ \\
\hline H9A & 0.637379 & 0.497195 & 0.792729 & $0.076^{\star}$ \\
\hline H9B & 0.565612 & 0.493921 & 0.904139 & $0.076^{\star}$ \\
\hline $\mathrm{H} 9 \mathrm{C}$ & 0.582649 & 0.609285 & 0.818803 & $0.076^{\star}$ \\
\hline C10 & $0.4256(3)$ & $0.3477(3)$ & $0.8287(3)$ & $0.0381(9)$ \\
\hline C11 & $0.3172(2)$ & $0.5470(2)$ & $0.8893(2)$ & $0.0493(9)$ \\
\hline $\mathrm{H} 11 \mathrm{~A}$ & 0.322983 & 0.627191 & 0.881290 & $0.074^{\star}$ \\
\hline H11B & 0.316209 & 0.506297 & 0.958212 & $0.074^{\star}$ \\
\hline $\mathrm{H} 11 \mathrm{C}$ & 0.247123 & 0.531495 & 0.874586 & $0.074^{\star}$ \\
\hline C12 & $0.4494(3)$ & $0.5633(2)$ & $0.6677(2)$ & $0.0252(7)$ \\
\hline C13 & $0.4215(2)$ & $0.8994(3)$ & $0.7771(2)$ & $0.0504(9)$ \\
\hline H13A & 0.406374 & 0.825660 & 0.771332 & $0.076^{\star}$ \\
\hline H13B & 0.502816 & 0.907240 & 0.769110 & $0.076^{\star}$ \\
\hline $\mathrm{H} 13 \mathrm{C}$ & 0.397585 & 0.957191 & 0.724931 & $0.076^{\star}$ \\
\hline C14 & $0.3986(2)$ & $0.8124(2)$ & $0.9984(2)$ & $0.0479(9)$ \\
\hline H14A & 0.367291 & 0.827412 & 1.063787 & $0.072^{\star}$ \\
\hline H14B & 0.481356 & 0.818753 & 0.980297 & $0.072^{\star}$ \\
\hline $\mathrm{H} 14 \mathrm{C}$ & 0.377452 & 0.736785 & 1.001643 & $0.072^{\star}$ \\
\hline C15 & $0.3748(3)$ & $1.0572(3)$ & $0.9052(4)$ & $0.0507(10)$ \\
\hline C16 & $0.1862(2)$ & $0.9023(3)$ & $0.9296(2)$ & $0.0256(7)$ \\
\hline C17 & $0.0098(4)$ & $0.7582(3)$ & $0.8129(4)$ & $0.0511(11)$ \\
\hline C18 & $-0.0888(3)$ & $0.9110(2)$ & $0.65977(19)$ & $0.0503(9)$ \\
\hline $\mathrm{H} 18 \mathrm{~A}$ & -0.152251 & 0.959645 & 0.645976 & $0.075^{\star}$ \\
\hline
\end{tabular}

Table 2 (continued)

\begin{tabular}{|c|c|c|c|c|}
\hline Atom & $x$ & $y$ & $z$ & $U_{\text {iso }} * / U_{\text {eq }}$ \\
\hline H18B & -0.087396 & 0.846539 & 0.632850 & $0.075^{\star}$ \\
\hline $\mathrm{H} 18 \mathrm{C}$ & -0.017573 & 0.952593 & 0.628081 & $0.075^{\star}$ \\
\hline C19 & $-0.2435(3)$ & $0.7864(3)$ & $0.8560(2)$ & $0.0712(12)$ \\
\hline H19A & -0.249493 & 0.751569 & 0.927031 & $0.107^{\star}$ \\
\hline H19B & -0.247299 & 0.728735 & 0.822322 & $0.107^{\star}$ \\
\hline $\mathrm{H} 19 \mathrm{C}$ & -0.306025 & 0.839063 & 0.849288 & $0.107^{\star}$ \\
\hline $\mathrm{C} 20$ & $-0.0962(3)$ & $0.9714(3)$ & $0.8602(2)$ & $0.0292(8)$ \\
\hline C21 & $0.0482(2)$ & $0.5724(2)$ & $1.08196(19)$ & $0.0442(9)$ \\
\hline $\mathrm{H} 21 \mathrm{~A}$ & -0.013782 & 0.546051 & 1.062491 & $0.066^{\star}$ \\
\hline $\mathrm{H} 21 \mathrm{~B}$ & 0.086771 & 0.508278 & 1.116130 & $0.066^{\star}$ \\
\hline $\mathrm{H} 21 \mathrm{C}$ & 0.102189 & 0.615383 & 1.022134 & $0.066^{*}$ \\
\hline C22 & $-0.1004(2)$ & $0.5710(2)$ & $1.28393(19)$ & $0.0462(9)$ \\
\hline $\mathrm{H} 22 \mathrm{~A}$ & -0.128958 & 0.614211 & 1.333060 & 0.069 * \\
\hline H22B & -0.055402 & 0.508184 & 1.311474 & $0.069^{\star}$ \\
\hline $\mathrm{H} 22 \mathrm{C}$ & -0.164314 & 0.542780 & 1.268507 & $0.069^{\star}$ \\
\hline $\mathrm{C} 23$ & $0.1118(3)$ & $0.7057(3)$ & $1.2036(3)$ & $0.0411(9)$ \\
\hline C24 & $-0.0763(3)$ & $0.7899(3)$ & $020(2)$ & $0.0323(8)$ \\
\hline $\mathrm{H} 3 \mathrm{~A}$ & $0.267(2)$ & $0.763(2)$ & $0.5910(18)$ & $0.028(9)^{\star}$ \\
\hline H3B & $0.251(2)$ & $0.877(2)$ & $0.5289(18)$ & $0.049(9)^{\star}$ \\
\hline $\mathrm{H} 3 \mathrm{C}$ & $0.366(2)$ & $0.829(2)$ & $0.513(2)$ & $0.053(12)^{\star}$ \\
\hline $\mathrm{H} 4 \mathrm{~A}$ & $0.3146(18)$ & $0.5963(17)$ & $0.3829(16)$ & $0.019(7)^{\star}$ \\
\hline $\mathrm{H} 4 \mathrm{~B}$ & $0.288(2)$ & $0.564(2)$ & $0.4884(18)$ & $0.035(9)^{\star}$ \\
\hline $\mathrm{H} 5 \mathrm{~A}$ & $0.807(2)$ & $0.624(2)$ & $0.530(2)$ & $0.052(10)^{\star}$ \\
\hline H5B & $0.718(2)$ & $0.647(2)$ & $0.6176(18)$ & $0.027(8)^{\star}$ \\
\hline $\mathrm{H} 5 \mathrm{C}$ & $0.816(2)$ & $0.719(2)$ & $0.573(2)$ & $0.052(10)^{\star}$ \\
\hline $\mathrm{H} 8 \mathrm{~A}$ & $0.5760(19)$ & $0.7528(19)$ & $0.3843(16)$ & $0.020(8)^{\star}$ \\
\hline H8B & $0.652(2)$ & $0.675(2)$ & $0.3840(17)$ & $0.023(9)^{\star}$ \\
\hline $\mathrm{H} 10 \mathrm{~A}$ & $0.490(2)$ & $0.3135(19)$ & $0.7992(17)$ & $0.029(8)^{\star}$ \\
\hline $\mathrm{H} 10 \mathrm{~B}$ & $0.358(2)$ & $0.331(2)$ & $0.8088(19)$ & $0.051(10)^{*}$ \\
\hline $\mathrm{H} 10 \mathrm{C}$ & $0.417(2)$ & $0.314(2)$ & $0.894(2)$ & $0.051(10)^{\star}$ \\
\hline $\mathrm{H} 12 \mathrm{~A}$ & $0.3758(19)$ & $0.5622(18)$ & $0.6671(16)$ & $0.018(7)^{\star}$ \\
\hline H12B & $0.4624(19)$ & $0.6309(19)$ & $0.6607(17)$ & $0.019(7)^{\star}$ \\
\hline H15A & $0.350(2)$ & $1.066(2)$ & $0.969(2)$ & $0.048(10)^{\star}$ \\
\hline H15B & $0.350(2)$ & $1.113(2)$ & $0.859(2)$ & $0.052(11)^{\star}$ \\
\hline $\mathrm{H} 15 \mathrm{C}$ & $0.454(2)$ & $1.061(2)$ & $0.8953(18)$ & $0.039(9)^{\star}$ \\
\hline $\mathrm{H} 16 \mathrm{~A}$ & $0.1812(19)$ & $0.829(2)$ & $0.9320(16)$ & $0.027(7)$ * \\
\hline H16B & $0.173(2)$ & $0.9311(19)$ & $0.8708(17)$ & $0.028(8)^{\star}$ \\
\hline H17A & $0.081(3)$ & $0.792(3)$ & $0.786(2)$ & $0.059(13)^{\star}$ \\
\hline H17B & $0.003(2)$ & $0.704(2)$ & $0.7794(19)$ & $0.053(9)^{\star}$ \\
\hline $\mathrm{H} 17 \mathrm{C}$ & $0.004(3)$ & $0.720(2)$ & $0.875(2)$ & $0.058(12)^{\star}$ \\
\hline $\mathrm{H} 20 \mathrm{~A}$ & $-0.158(2)$ & $1.0121(18)$ & $0.8559(16)$ & $0.023(7)^{\star}$ \\
\hline $\mathrm{H} 20 \mathrm{~B}$ & $-0.047(2)$ & 1.011(2) & $0.816(2)$ & $0.044(11)^{\star}$ \\
\hline $\mathrm{H} 23 \mathrm{~A}$ & $0.140(2)$ & $0.645(2)$ & $1.2394(19)$ & $0.044(9)^{\star}$ \\
\hline H23B & $0.171(2)$ & $0.731(2)$ & $1.1512(18)$ & $0.033(9)^{\star}$ \\
\hline $\mathrm{H} 23 \mathrm{C}$ & $0.092(2)$ & $0.763(2)$ & $1.242(2)$ & $0.063(10)^{\star}$ \\
\hline $\mathrm{H} 24 \mathrm{~A}$ & $-0.1198(19)$ & $0.8160(18)$ & $1.1474(16)$ & $0.025(8)^{\star}$ \\
\hline H24B & $-0.126(2)$ & $0.7634(19)$ & $1.0807(17)$ & $0.021(8)^{\star}$ \\
\hline
\end{tabular}

173(2) K using graphite-monochromated Mo $K \alpha$ radiation $(\lambda=0.71073 \AA)$. The crystal structure was solved with direct methods (SHELXS-97 [2, 3]) and refined against $F^{2}$ with the full-matrix least-squares method (SHELXL-2018/3 [2, 4, 5]). A multi-scan absorption correction using the implemented CrysAlis RED program was employed [1]. The non-hydrogen 
atoms were refined using anisotropic displacement parameters. The methylene hydrogen atoms and the methyl hydrogen atoms bonded to $\mathrm{C} 3, \mathrm{C} 5, \mathrm{C} 10, \mathrm{C} 15, \mathrm{C} 17$, and $\mathrm{C} 23$ were located on the difference Fourier map and refined independently. All other hydrogen atoms were placed in idealized geometric positions and each was assigned a fixed isotropic displacement parameter based on a riding-model. The $U_{\text {iso }}$ values for the methyl hydrogen atoms were set to $1.5 U_{\text {eq }}$ of the parent carbon atoms with the methyl $\mathrm{C}-\mathrm{H}$ distances constrained to $0.98 \AA$. The visualization of the molecular structure shown in the figure was performed using Mercury 4.1.0 [6].

\section{Discussion}

(Trimethylsilyl)methyllithium $\left(\mathrm{LiCH}_{2} \mathrm{SiMe}_{3}\right)$ is a commonly used reagent for silyl transfer reactions $[9,10]$, for halogenlithium exchange [11], and was recently used for the deprotonation of toluene to form pure crystalline benzyllithium [12]. The crystal structure of $\mathrm{LiCH}_{2} \mathrm{SiMe}_{3}$ was first reported by Tecle et al. as a hexameric aggregate in the monoclinic crystal system, space group $P 2_{1} / n$ [13]. In addition to the donor base-free molecular structure of $\left[\mathrm{LiCH}_{2} \mathrm{SiMe}_{3}\right]_{6}$, only a few deaggregated structures of (trimethylsilyl)methyllithium have been reported to date [14-17]. $\quad N, N, N^{\prime}, N^{\prime}$-Tetramethylethylenediamine (TMEDA) and (-)-sparteine form the dimers $\left[(\mathrm{TMEDA}) \cdot \mathrm{LiCH}_{2} \mathrm{SiMe}_{3}\right]_{2}$ and $\left[\{\text {-sparteine }\} \cdot \mathrm{LiCH}_{2} \mathrm{SiMe}_{3}\right]_{2}$, whereas $N, N, N^{\prime}, N^{\prime \prime}, N^{\prime \prime}$ pentamethyldiethylenetriamine (PMDETA) forms the monomeric [(PMDETA) $\cdot \mathrm{LiCH}_{2} \mathrm{SiMe}_{3}$ ] adduct [14]. Diethyl ether and tert-butylmethyl ether deaggregate the hexameric parent compound $\left[\mathrm{LiCH}_{2} \mathrm{SiMe}_{3}\right]_{6}$ to the unsymmetrically coordinated tetramers $\left[\left(\mathrm{Et}_{2} \mathrm{O}\right)_{2} \cdot\left(\mathrm{LiCH}_{2} \mathrm{SiMe}_{3}\right)_{4}\right]$ and $\left[(t \mathrm{BuOMe})_{2} \cdot\left(\mathrm{LiCH}_{2} \mathrm{SiMe}_{3}\right)_{4}\right]$, while dimethoxyethane (DME) forms the dimer $\left[(\mathrm{DME}) \cdot\left(\mathrm{LiCH}_{2} \mathrm{SiMe}_{3}\right)\right]_{2}$ [15]. In 2015, we reported on a dimeric (trimethylsilyl)methyllithium species using a chiral dimethoxysilane as chelating ligand [16]. A recently published article describes a monomeric complex consisting of (trimethylsilyl)methyllithium and the bidentate chiral ligand $(1 R, 2 R)-N, N, N^{\prime}, N^{\prime}$-tetramethylcyclohexane1,2-diamine together with a symmetrically and an unsymmetrically coordinated dimeric quinuclidine adduct of $\left[\mathrm{LiCH}_{2} \mathrm{SiMe}_{3}\right]_{6}[17]$.

The molecular structure of the title compound presented herein is the second polymorph found for (trimethylsilyl)methyllithium. The hexamer $\left[\mathrm{LiCH}_{2} \mathrm{SiMe}_{3}\right]_{6}$ crystallized in the triclinic crystal system, space group $P \overline{1}$. The asymmetric unit contains two crystallographically independent half aggregates, each being arranged around a center of inversion (cf. the figure). The lithium atoms of each hexamer are threefold coordinated and build a trigonal antiprismatic geometry with average $\mathrm{Li} \cdot$. L Li distances of $3.22 \AA$ within the triangular base and $2.45 \AA$ A between neighboring lithium atoms when following the lithium core in a zig-zag course. These distances are similar to those in monoclinic $\left[\mathrm{LiCH}_{2} \mathrm{SiMe}_{3}\right]_{6}(2.45 \AA$ and $3.18 \AA$ ) [13]. The bond lengths between the silicon and methylene carbon atoms differ from 1.839(3) $\AA$ (C4-Si1) to 1.868(3) $\AA$ (C24-Si6). The carbanionic carbon atoms are coordinated to three lithium centers leading to a $\left[\mathrm{Li}_{6} \mathrm{C}_{6}\right]$ core that features two $\left[\mathrm{Li}_{3} \mathrm{C}_{3}\right]$ six-membered folded chairs arranged in parallel with alternating $\mathrm{Li}-\mathrm{C}$ bond lengths ranging from 2.139(5) $\AA$ (C4-Li3) to 2.280(5) $\AA$ (C20-Li4\#2 and Li4-C20\#2; \#2 = - $x$, $-y+2,-z+2)$, which are similar to those found in monoclinic (trimethylsilyl)methyllithium (2.15 A to 2.29 ̊) [13].

Lithium-hydrogen interactions in organolithium compounds and their possible contribution to the aggregate formation have been the subject of discussion for a long time $[13,18,19]$. Similar to the monoclinic hexamer $\left[\mathrm{LiCH}_{2} \mathrm{SiMe}_{3}\right]_{6}$, the here described triclinic polymorph shows short $\mathrm{Li} \cdots \mathrm{H}$ distances ranging from $2.014 \AA$ (Li1-H4B) to $2.218 \AA$ (Li2H8A) for the carbanionic methylene groups and from $2.264 \AA$ (Li5-H17C) to $2.553 \AA$ (Li5-H17A) for the C3, C5, C10, C15, C17, and $\mathrm{C} 23$ methyl groups that are oriented towards a lithium atom. The shortest $\mathrm{Li} \cdots \mathrm{H}$ distance found in the monoclinic polymorph amounts to 2.03(6) Å [13].

Acknowledgements: The Elite Network of Bavaria (ENB), the Bavarian State Ministry of Science and the Arts (StMWK), and the University of Regensburg are gratefully acknowledged for financial support.

\section{References}

1. Oxford Diffraction Ltd., CrysAlis CCD and CrysAlis RED, Abingdon, England (2008).

2. Sheldrick, G. M.: A short history of SHELX. Acta Crystallogr. A64 (2008) 112-122.

3. Sheldrick, G. M.: SHELXS-97, a program for the solution of crystal structures. Universität Göttingen, Göttingen, Germany (1997).

4. Sheldrick, G. M.: Crystal structure refinement with SHELXL. Acta Crystallogr. C71 (2015) 3-8.

5. Sheldrick, G. M.: SHELXL-2018. Universität Göttingen, Göttingen, Germany (2018).

6. Macrae, C. F.; Edgington, P. R.; McCabe, P.; Pidcock, E.; Shields, G. P.; Taylor, R.; Towler, M.; van de Streek, J.: Mercury: visualization and analysis of crystal structures. J. Appl. Cryst. 39 (2006) 453-457.

7. Westrip, S. P.: pubICIF: software for editing, validating and formatting crystallographic information files. J. Appl. Cryst. 43 (2010) 920-925.

8. Farrugia, L. J.: WinGX and ORTEP for Windows: an update. J. Appl. Cryst. 45 (2012) 849-854.

9. Yu, J. M.; Teyssié, D.; Khalifa, R. B.; Boileau, S.: Anionic polymerization and copolymerization of cyclosiloxanes initiated by trimethylsilylmethyllithium. Polym. Bull. 32 (1994) 35-40.

10. Skvortsov, G. G.; Fukin, G. K.; Trifonov, A. A.; Noor, A.; Döring, C.; Kempe, R.: Intramolecular $\left(\mathrm{sp}^{3}\right.$-hybridized) $\mathrm{C}-\mathrm{H}$ 
activation: yttrium alkyls versus transient yttrium hydrides. Organometallics 26 (2007) 5770-5773.

11. Doudouh, A.; Woltermann, C.; Gros, P. C.: $\mathrm{TMSCH}_{2} \mathrm{Li}$ and $\mathrm{TMSCH}_{2} \mathrm{Li}$-LiDMAE: efficient reagents for noncryogenic halogen-lithium exchange in bromopyridines. J. Org. Chem. 72 (2007) 4978-4980.

12. Tatic, T.; Hermann, S.; John, M.; Loquet, A.; Lange, A.; Stalke, D.: Pure $\alpha$-metallated benzyllithium from a singlecrystal-to-single-crystal transition. Angew. Chem. Int. Ed. 50 (2011) 6666-6669; Angew. Chem. 123 (2011) 6796-6799.

13. Tecle, B.; Maqsudur Rahman, A. F. M.; Oliver, J. P.: X-ray crystal structure of trimethylsilylmethyllithium. J. Organomet. Chem. 317 (1986) 267-275.

14. Tatic, T.; Ott, H.; Stalke, D.: Deaggregation of trimethylsilylmethyllithium. Eur. J. Inorg. Chem. 24 (2008) 3765-3768.

15. Tatic, T.; Meindl, K.; Henn, J.; Pandey, S. K.; Stalke, D.: The first asymmetric organolithium tetramers with simple ether donor bases. Chem. Commun. 46 (2010) 4562-4564.

16. Bauer, J. O.; Strohmann, C.: Stereocontrol in nucleophilic substitution reactions at silicon: the role of permutation in generating silicon-centered chirality. J. Am. Chem. Soc. 137 (2015) 4304-4307.

17. Knauer, L.; Wattenberg, J.; Kroesen, U.; Strohmann, C.: The smaller, the better? How the aggregate size affects the reactivity of (trimethylsilyl)methyllithium. Dalton Trans. 48 (2019) 11285-11291.

18. Craubner, I.: Beiträge zur Struktur und Assoziatbindung des hexameren $n$-Butyl-lithium. Z. Phys. Chem. 51 (1966) 225-233.

19. Zerger, R.; Rhine, W.; Stucky, G.: Stereochemistry of polynuclear compounds of the main group elements. The bonding and the effect of metal-hydrogen-carbon interactions in the molecular structure of cyclohexyllithium, a hexameric organolithium compound. J. Am. Chem. Soc. 96 (1974) 6048-6055. 